\title{
Shear-Induced Microporous Nanocomposite Epoxy Thermosets (MiNET)
}

Molla Hasan, Yogin Patel, Arielle R. Gamboa, Michael J. Grzenda, Valeria Saro-Cortes, Vivek

Mhatre, Jonathan P. Singer

Prof. Molla Hasan

Inamori School of Engineering, Alfred University, Alfred, NY 14802, USA

Department of Mechanical \& Aerospace Engineering, Rutgers University, Piscataway, NJ

08854, USA

Email: hasanm@alfred.edu

Yogin Patel

Department of Mechanical \& Aerospace Engineering, Rutgers University, Piscataway, NJ 08854, USA

Arielle R. Gamboa

Department of Mechanical \& Aerospace Engineering, Rutgers University, Piscataway, NJ 08854, USA

Michael J. Grzenda

Department of Mechanical \& Aerospace Engineering, Rutgers University, Piscataway, NJ 08854, USA

Valeria Saro-Cortes

Department of Mechanical \& Aerospace Engineering, Rutgers University, Piscataway, NJ 08854, USA

Vivek Mhatre

Department of Mechanical \& Aerospace Engineering, Rutgers University, Piscataway, NJ 08854, USA

Prof. Jonathan P. Singer

Department of Mechanical \& Aerospace Engineering, Rutgers University, Piscataway, NJ 08854, USA

Email: jonathan.singer@rutgers.edu

\begin{abstract}
To create microporous nanocomposite epoxy thermosets (MiNET), a mixing pathway is demonstrated in which bicontinuous inferfacially jammed emulsion gels (bijels) like viscous fluid is kinetically trapped by high shear mixing of immiscible liquids, surfactant, and nanoparticles. The MiNETs are prepared from common ingredients, that are widely employed in
\end{abstract}


industry, including epoxy resin, vegetable oil, epoxidized soybean oil, and different types of nanoparticles such as silica, activated carbon, alumina, and zinc oxide. MiNETs prepared by the presented route are processed at ambient conditions and exhibit low shrinkage (less than 2\%). Furthermore, they are suitable to erect macro- to microscale structures with high precision and various porosity. The interconnected porous architecture of MiNET is even preserved in microscale features and thus ensures the mass transport in microstructures. With facile processability and tunability of pore sizes in a wide range ( $\sim 100 \mathrm{~nm}$ to few microns), the proposed route overcomes the two major roadblocks - difficulty in fabrication and large domain size (on the order of $5 \mu \mathrm{m}$ or larger) - of bijels-like materials to apply in catalysis, energy storage, and molecular encapsulation.

\section{Introduction:}

Porous materials with tunable surface to volume ratio and chemistry have numerous applications, including tissue engineering, ${ }^{[1]}$ catalysis,${ }^{[2]}$ smart filtering, ${ }^{[3,4]}$, fuel cells, ${ }^{[5]}$ and sensors..${ }^{[6,7]}$ These materials are also essential to unlock the physics of mass transport phenomena in porous media. ${ }^{[7,8]}$ To synthesize the porous materials in the aforementioned applications and studies, numerous methods have been developed, for example metal-organic frameworks (MOFs), solgel, particles sintering, and emulsion templating. MOFs excel at gas separations, but are not appropriate for liquid transport due to their extremely small pores (less than $2 \mathrm{~nm}$ ). ${ }^{[9]}$ Sol-gel processing, ${ }^{[10]}$ particles sintering, ${ }^{[11]}$ and emulsion templating ${ }^{[12]}$ produce micro- to meso-scale porous monoliths, which are suitable for mass transport. However, these methods for making porous monoliths require high-temperature treatments and/or result in volume loss/shrinkage that can lead to fracture. Moreover, the methods lack systematic variation of pore sizes from nano to micro. Bicontinuous interfacially jammed emulsion gels, or bijels, are an emerging category of 
porous materials with interconnected pores and tunable porosity and chemistry ${ }^{[13,14]}$ Bijels are particle-stabilized emulsions in which two inter-penetrating continuous phases exist. ${ }^{[15,16]}$ The $3 \mathrm{D}$ porous morphology of bijel is attractive because of the large and tunable interfacial area, ${ }^{[17-19]}$ simultaneous transport, ${ }^{[20-22]}$ and improved mechanical properties. ${ }^{[23,24]}$ In bijels, the size of tortuous channel and the size of the pores are possible to control through the volume fraction of particles and the particle size, respectively. In all cases, the pore size is much larger than the particle employed because it scales as $a / \varnothing$ (where $a$ and $\varnothing$ are radius and volume fraction of the particles) ${ }^{[25]}$ for instance, silica microspheres with diameter around $750 \mathrm{~nm}$ provide bicontinuous porous template with pore dimensions ranging from 10 to over $100 \mu \mathrm{m} .{ }^{[26]}$ Given bicontinuous spinodal morphology allows intimate contact between two immiscible liquids, bijels have been envisioned as microfluidic chamber for crossflow micro-reactions ${ }^{[14,25]}$ and as transporter for controlled release of encapsulated materials. ${ }^{[27]}$ Owing to their nonsequestered nature of the materials phases and advantageous morphology, other potential applications of bijels include, use as a template for catalysts, electrodes for batteries, hierarchically porous materials, and artificial scaffolds for tissue engineering. ${ }^{[26,28,29]}$ Two methods have been reported to create bijels. The first method leverages the phase separation of partially miscible liquid via spinodal decomposition to create a cocontinuous fluid network that is stabilized by particles. ${ }^{[14,}$ ${ }^{27]}$ The bicontinuous network is used as a template in which one fluid phase is polymerized and another one is drained out to create porous scaffold. ${ }^{[28]}$ The second method follows a simple mixing protocol of two partly immiscible liquids along with a surfactant and particles, where instead of spinodal decomposition, sub-micrometer domain is formed via homogenization. ${ }^{[30,31]}$ Advantages of mixing method over the template-assistance method include: (1) neutrally wetting particles are not required (2) the choice of liquids is not limited. While both the methods provide 
interconnected permeation path, they require delicately prepared colloids, exhibit poor thermal stability (in case of template-assistance method), and are not suitable for molding the structures with accuracy due to its high fluidity (low viscosity) at the beginning of curing. Moreover, shrinkage of the bijel materials may pose a greater challenge to integrate them into other devices (such as microfluidic) and replicate structures precisely. Hence, there is a need for a means to make porous materials that can be processed in ambient conditions with reduced materials loss, tunable porosity, and high moldability. Herein, we describe a general approach of making a bijellike structure using high-shear mixing of two immiscible phases (here epoxy-phenolic resin and a vegetable oil porogen), surfactant, and nanoparticles. Due to the curing of the epoxy, the prepared monolith can be turned to porous material by removing the porogen from the bulk. Because the bijel-inspired monolith consists nanoparticles and has micro-pores, we name it microporous nanocomposite epoxy thermoset (MiNET). We demonstrate MiNET is moldable to desired structure with minimum shrinkage and the porous structure enables mass transport, which could be employed for interfacial catalysis for diverse chemical reactions and to study the flow physics of complex porous materials such as soils, rocks, and tissues.

\section{Results and Discussion}

Epoxy-phenolic resins, which are valued for their ease of processing into various shapes and coating thicknesses, have been widely used as base materials for a wide variety of applications, such as integrated circuit packages, laminating resins, adhesives, binders, surface coatings, and impregnants. ${ }^{[32]}$ We use epoxy resin (bisphenol A/epichlorohydrin derived liquid epoxy resin with versamid crosslinker) as a major constituent of MiNET. To make MiNET, we begin with a bicontinuous mixture created by high shear (Figure 1) comprised of immiscible mixtures of two fluids: epoxy and canola oil (porogen), stabilized by a surfactant epoxidized soybean oil (ESO) 
(Figure 1a), which also improves the interfacial bonding between the epoxy and the canola oil. Since the proportion of this mixture are not specifically optimized, it is likely that it would coarsen into a stabilized emulsion of droplets with size determined by the amount of surfactant. However, by adding nanoparticles interfacial jamming occurs (Figure $l \mathrm{~b}$ ), which arrests the fluids in a bicontinuous configuration far from the demixing point of the liquids. Due to their size induced higher diffusion coefficient, particles less than $5 \mu \mathrm{m}$ can adsorb onto the liquidliquid interface more rapidly. ${ }^{[33,34]}$ During the evolution (polymerization and crosslinking) of the emulsion, the configuration maintains its inter-penetrating continuous phases as the energy cost of removing these particles from the interface is typically extremely large and can exceed thermal energy by several orders of magnitude depending on the size of the particles, the liquidliquid, and liquid-particle surface tensions, and the line tension of the three-phase contact line. ${ }^{[33,}$ ${ }^{35]}$ Canola oil does not take part in the polymerization, but the epoxy-rich phase solidifies due to the crosslinking of its monomer (bisphenol A/epichlorohydrin) and hardener (Versamid 125). After the completion of curing, canola oil is removed by a solvent (acetone), resulting in an interconnected porous network throughout the epoxy-nanocomposite matrix (Figure 1c). Activated carbon nanoparticles $(100 \mathrm{~nm})$ based MiNET and its surface and bulk morphology are shown in Figure $1 \mathrm{~d}-\mathrm{e}$. The porous nature of MiNETs makes them fragile. Moreover, structures formed of hard nanoparticles, such as silica and activated carbon, are brittle in nature, and hence, the porous materials consisting of such nanoparticles lack the structural integrity and abrade easily. ESO can minimize the issue by acting as a plasticizer in addition to its surfactant role, and our rheological studies corroborate the dual role of ESO in MiNET preparation. Figure 1g shows the curing kinetics of activated carbon based MiNET with various ESO contents. At a low loading of ESO $(\leq 6 \%)$ it does not affect in the curing due to interfacial segregation, but 
increases the storage modulus due to reduced emulsion domain size ${ }^{[36]}$ conversely, ESO at high loadings ( $\geq 8 \%$ ) hinders the curing kinetics and reduces modulus. Although tailored polyamide such as dicarboxyl terminated polyamide and diamino ended polyamide can bond ESO moiety, polyamide itself cannot break the three-atom ring of epoxide of ESO. ${ }^{[37,38]}$ The unaltered epoxides and hydrocarbons of ESO when present in a small fraction in the epoxy-canola oil emulsion, however, strengthen the interfacial bonding of the two immiscible fluids and as a result, the storage modulus, $G^{\prime}$ of the MiNETs increases. As the ESO amount exceeds the critical fraction limit (more than 6\%), the chain reaction of polymerization retards, and the excess amount of uncured ESO reduces the crosslinking density of polymer matrix. As a result, the flexible property of the MiNET increases that is marked by the decrease of $G^{\prime}$.

This method is compatible with a variety of other micro- and nanoparticles, such as alumina $\left(\mathrm{Al}_{2} \mathrm{O}_{3}\right)$, super activated carbon, zinc oxide $(\mathrm{ZnO})$, chitosan, graphene, graphite flakes, silica, silicone, and aluminum as shown in Figure 2 and Figure SI1 of Supporting Information (SI). However, the volume fraction of nanoparticles to make MiNETs and curing kinetics of the polymer composites differ with the type of nanoparticles as some particles promote polymerization and other decelerate chain reactions. Using functional nanoparticles such as $\mathrm{ZnO}$ and $\mathrm{Al}_{2} \mathrm{O}_{3}$, MiNET could easily be deployed in applications such as anti-corrosive coatings ${ }^{[39]}$ and dielectric materials ${ }^{[40,41]}$, while MiNET made from silicone particles are mechanically flexible (Figure SI1b,c). The compositions of the MiNETs using the particles shown in Figure 2 are available in Table SI1. The length scale of the pores is determined by the selection of particles' size and volume fraction of the porogen. SEM images in Figure 2g-i show how the pore size of the silica-based monolith changes with particle sizes. Sample made with $60 \mathrm{~nm}$ silica 
particles yields smaller pores compared to samples prepared by $400 \mathrm{~nm}$ and $1 \mu \mathrm{m}$. Figure $2 \mathrm{~g}-\mathrm{i}$ also reveal pores are uniformly distributed throughout the surface and are interconnected.

To understand the effect of particle size on porosity, we have conducted mercury intrusion porosimetry of silica-based five different MiNETs samples. The average size of the silica particles used in these samples ranges from $60 \mathrm{~nm}$ to $45 \mu \mathrm{m}$. The ratio of particles and epoxy of these MiNETs samples is 9.375:1. The differential pore size distribution of these samples are shown in Figure 3. The peak of the curves shifts from left to the right as the particle size increases, which implies mean pore diameter, $d$ of the MiNETs can be governed by altering the particle size. Only the curve of Silica $60 \mathrm{~nm}$ sample exhibits a bimodal nature in which two peaks are located at the diameter of $50 \mathrm{~nm}$ and $85 \mathrm{~nm}$. The dual pore-structures is stemmed from the formation of intra-aggregate and inter-aggregate pores in the monolith. Such bimodal distribution is common in rocks ${ }^{[42]}$ and soils ${ }^{[43]}$ and is also reported for porous composite materials ${ }^{[44]}$ and ceramics. ${ }^{[45]}$ It is also interesting to note that the peak location of all the samples except silica $45 \mu \mathrm{m}$ lies in the range of particle sizes for the powder, hence suggesting that the desire pore dimension is possible to achieve by selecting the appropriate size of the particles. Since pore size scale as $a / \varnothing$, high volume fraction of particles leads the pore size close to particle size. The inset of Figure 3 shows the reproducibility of the same peak location of three silica 60 $\mathrm{nm}$ samples. Indeed, the silica used in making the MiNET sample of silica $45 \mu \mathrm{m}$ has a broad particle size distribution and a significant fraction of the particles are sub-micron in size, which fills the macroscopic pores during the process and reduces the average pore diameter. Because the particles dictate the pore structures, the porosity of the MiNETs also varies with the size of particles. Smaller particles yield smaller pores, but higher porosity, as listed in Table 1. We have achieved around $60 \%$ porosity in MiNET using $60 \mathrm{~nm}$ silica particles and porosity greater than 
this may be possible by employing even smaller particles and optimizing the compositions, though this might also require higher shear.

In epoxy, when van der Waals distances between unreacted monomers are converted to covalent bonds upon polymerization, the resulting microscopic free volume loss causes shrinkage. ${ }^{[46]}$ Also, in porous materials, the shrinkage induced stress triggers the collapse of voids, exacerbating the volume loss of monoliths. The silica-based MiNETs contain less than $11 \%$ (by mass) of monomer and crosslinker in their composition, and as a result, the volumetric shrinkage is not significant. Moreover, the high mass fraction of nanoparticles in the polymer matrix maintains the structural integrity of pores by withstanding shrinkage stress. Hence, despite the high porosity, the shrinkage of the MiNETs is significantly low $(<2 \%)$ even after removing the porogen and drying (Table 1).

The high viscosity of MiNETs after mixing eases the processing of molding into macro to sub-micron components. Highly viscous uncured MiNETs deform under external load but retain the shape after releasing the load. In addition, the high viscous force can overcome the capillary pressure, and as a result, even sub-micron length features can be replicated precisely. The MiNET we developed can have a viscosity ranging from toothpaste-like to putty-like based on composition. Therefore, MiNETs are suitable for molding, extrusion, and embossing to make structures with different length scales. Figure 4a shows the replication of two macroscopic structures - hand skeleton and earth medallion - and an array of micro-pillars made of MiNETs by embossing, where uncured MiNET is pressed against a solid template with concave impression. Due to low shrinkage, the dimensional accuracy of any replicated parts by molding is high and the parts can copy the small details of the master templates. Also, the slight shrinkage can be a processing aid in acting as a mold release along with the surface oil layer. 
To employ any porous materials in mass transport applications or to be a representative porous media for fluid flow study, porous materials should have open-cell porosity that ensures mass transport. Although the SEM images in Figure 1 d,f show the pores (both on the surface and in bulk) of MiNET are interconnected, the morphology in the surface and the bulk can be different once the uncured MiNET is forced through a narrow opening. In the case of narrow opening (microscale), there is a possibility that nanoparticles' aggregation restricts the flow in such a way that only the liquid phase (epoxy and porogen) fills into the narrow cavity. Because the porous materials need to integrate into the microchannel of the microfluidic device for masstransport applications, it is essential to characterize the porous architecture of the materials when it is crafted to a micro-scale feature. To do so, we made micropillars through molding and then sliced one of the pillars in a vertical direction using the focused ion beam (Figure 4b). It is evident from the SEM images (Figure 4b) that the bicontinuous porous structure is similar in the base and the pillar. We can assume from the SEM image that as long as the cross-section of channels is larger than the particle size, viscous MiNET can flow like Poiseuille flow, and as a result, the composition remains homogenous during filling of the microholes/microchannels. We also performed the infiltration test of the porous materials using ethylene glycol doped with a fluorescent dye, rhodamine B. In this test, dyed ethylene glycol (DEG) was infiltrated from the bottom of the MiNET substrate, which has micropillars on it. This DEG transported through the base, reached the tip of micropillar, and changed the color of the pillar to pink, as shown in the confocal image (Figure 4c). On the other hand, the top part of another pillar which was broken halfway and rested on the substrate remained blue because it was not infused with DEG (Figure 4c). This test proves that MiNETs can transport fluid if the media is continuous.

\section{Conclusions:}


In summary, we present a bijel-based mixing method to make MiNET that is scalable, suitable for mass transport, and exhibits low shrinkage. The method is a simple mixing of ternary liquids and nanoparticles and is processed at ambient conditions without using the stringent and tedious protocol. We unlock the potential of ESO as a surfactant besides its role as a plasticizer. Compared to the conventional methods, our method is compatible with a large variety of economic micro- and nanoparticles and apt for a pair of partially miscible liquids, opening the possibility of a wide range of immiscible and miscible liquids to make bijel. We demonstrate that making a designer porous material with various porosity and pore size (adjustable over an order of magnitude) is possible only by changing the particle size. Because of the moldability and low shrinkage, MiNETs are ideal to imitate macro- to microstructures with high precision. Moreover, MiNETs could be extended to 2D or 3D printing to erect complex architecture for the desired applications.

\section{Experimental Section}

All the materials were used as received. Activated carbon, super activated carbon, zinc oxide $(\mathrm{ZnO})$, alumina $\left(\mathrm{Al}_{2} \mathrm{O}_{3}\right)$, and aluminum nanoparticles were procured from Skyspring Nanomaterials. Silica micro- and nanoparticles were purchased from US-Nano. Chitosan and silicone microparticles (DOSIL ${ }^{\mathrm{TM}}$ EP-5500) were received from Sigma Aldrich and DOW Corporate, respectively. Difunctional bisphenol A/epichlorohydrin derived liquid epoxy resin $\left(\mathrm{EPON}^{\mathrm{TM}} 828\right)$ was used with polyamide curing agent (Versamid 125). The epoxy resin and polyamide curing agent, and epoxidized soybean oil (Vikoflex $\left.{ }^{\circledR} 7170\right)$ were supplied by SkyGeek, Gabriel Performance Products, and Arkema, respectively. Canola oil, ethylene glycol, and rhodamine B were procured from Sigma Aldrich. 
To prepare MiNET, epoxy resin, crosslinker, ESO, canola oil, and nanoparticles were mixed using speed-mixer (manufactured by FlackTek, Inc.) at $3000 \mathrm{rpm}$ for two minutes. The MiNET was cured at ambient condition for 16-24 hours (based on the compositions). To characterize the porosity, we used scanning electron microscope, mercury intrusion porosimeter, confocal microscope, and focused ion beam.

\section{Acknowledgements}

This work was supported by Espace Inc. A.R.G and V.S-C. additionally acknowledge support by NASA through the New Jersey Space Grant Consortium. M.H and M.J.G acknowledge support from Mistletoe Foundation and SubUAS Inc., respectively. The authors also like to thank Prof. Herbert Giesche (Alfred University) for helping in porosity measurement.
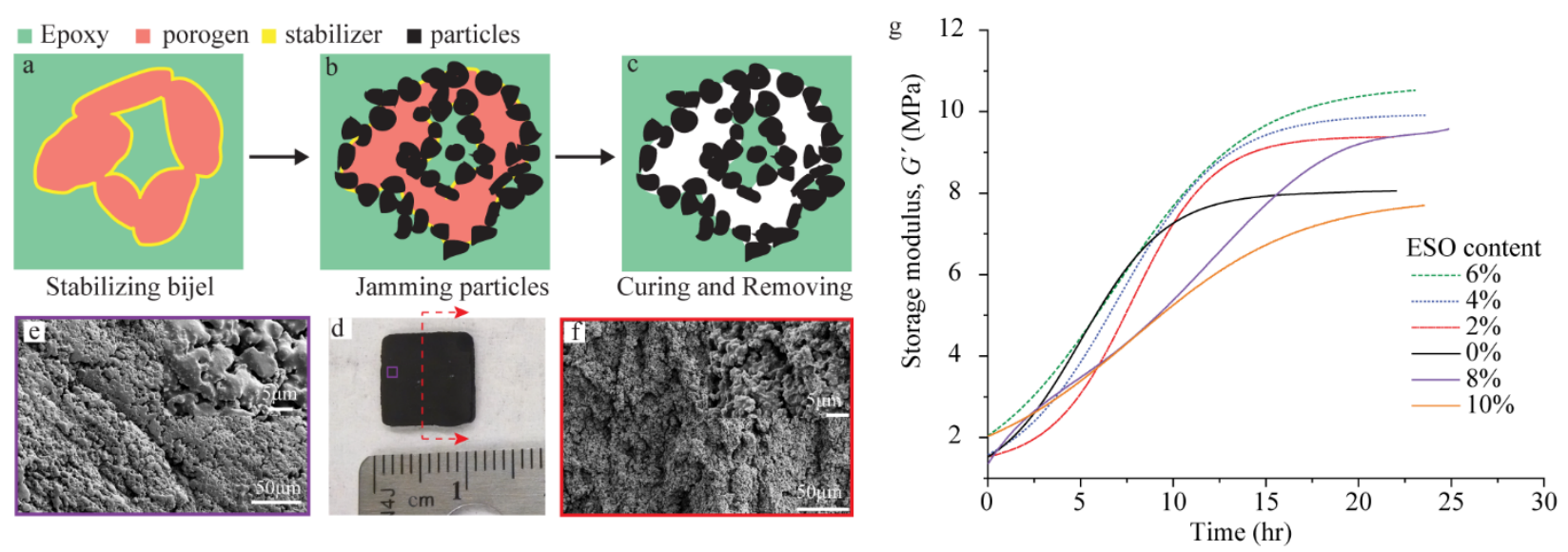

Figure 1: Schematic of the mechanism of bijel materials preparation: (a) forming and stabilizing of bicontinuous gel, (b) arresting the morphology by nanoparticles jamming, (c) creating interconnected porous structures by curing the epoxy phase and removing porogen. (d) Photo of activated carbon reinforced MiNET monolith, (e) SEM image of the monolith's surface, (f) Morphology of the monolith's bulk; to take this image, the monolith was broken into two pieces and the image of cross-section was taken by SEM. (g) Effect of ESO in the curing kinetics of activated carbon based MiNETs. The percentage of ESO is weight percentage. 

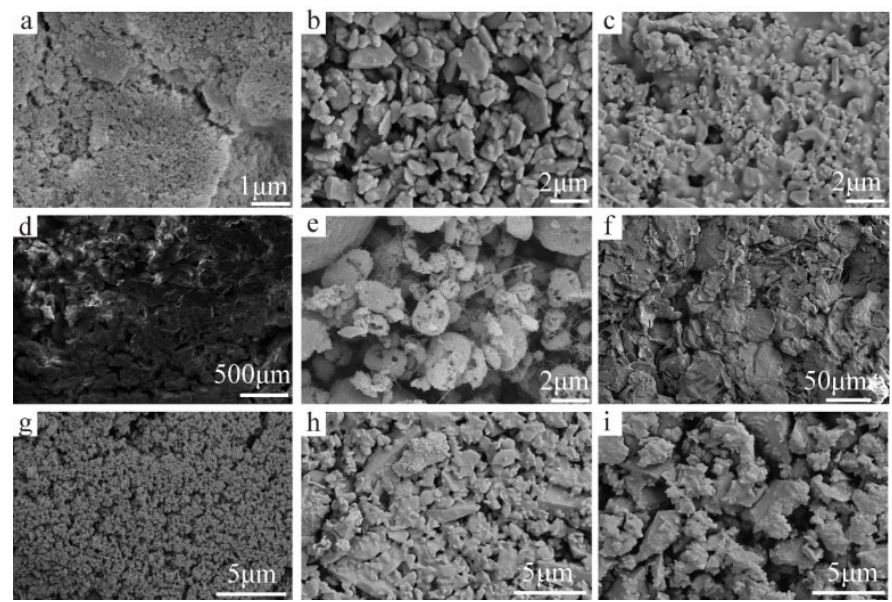

Figure 2: SEM images of different MiNETs made of:(a) $\mathrm{Al}_{2} \mathrm{O}_{3}$ (b) super activated carbon, (c) $\mathrm{ZnO}$, (d) chitosan, (e) graphene, (f) graphite flakes, (g) silica $60 \mathrm{~nm}$, (h) silica $400 \mathrm{~nm}$, and (i) silica $1 \mu \mathrm{m}$.

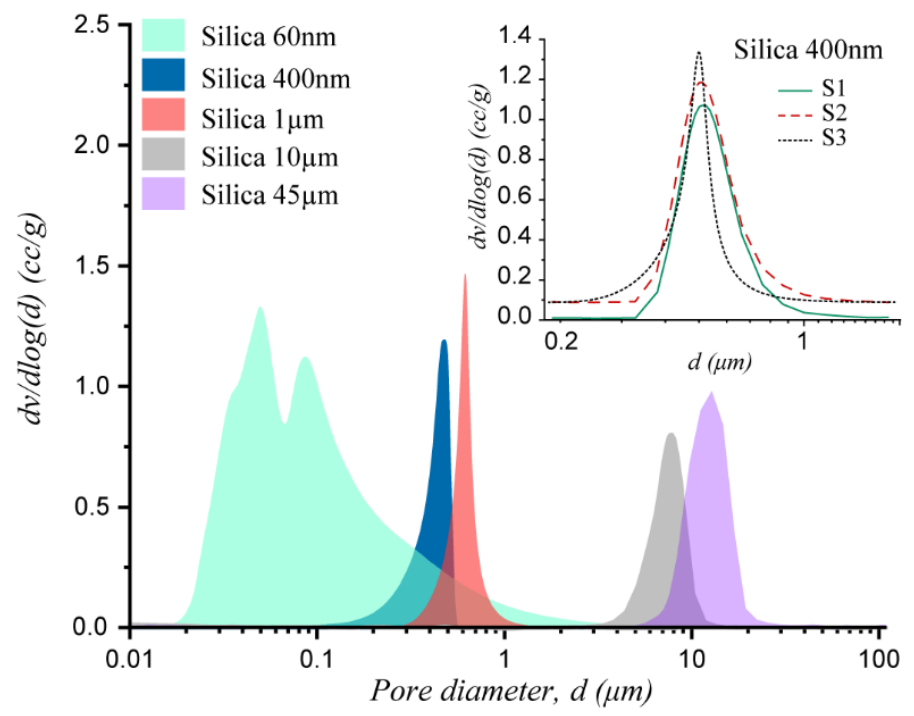

Figure 3: Differential pore size distribution of five MiNETs prepared using silica 60nm, 400nm, $1 \mu \mathrm{m}, 10 \mu \mathrm{m}$, and $45 \mu \mathrm{m}$ particles. (inset) Differential pore size distribution three MiNETs samples (S1, S2, and S3) made of silica $400 \mathrm{~nm}$ particles. The curves of S2 and S3 have been shifted to the vertical direction by 0.1 for better visibility. 


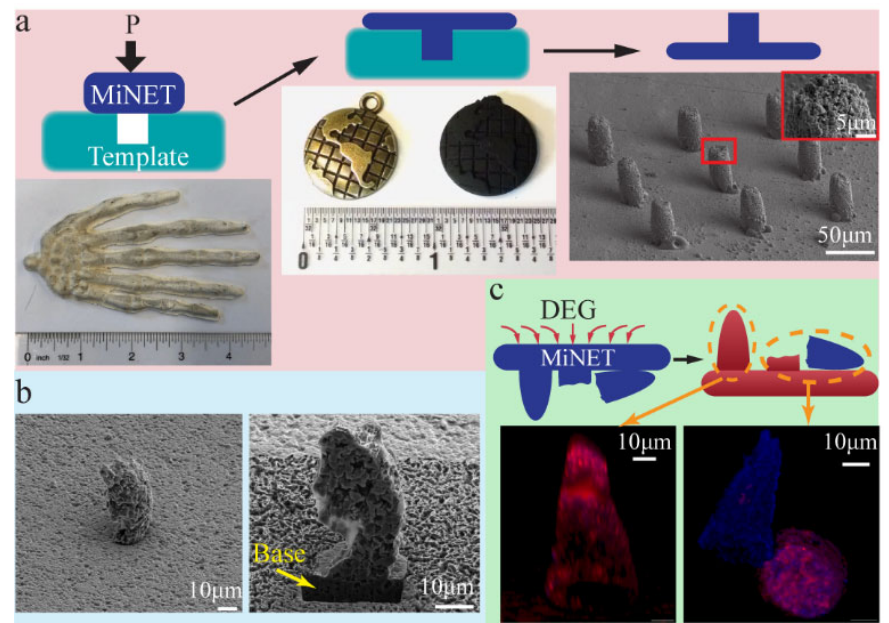

Figure 4:(a) Schematic of embossing of MiNET to make a structure, photo of hand skeleton and earth medallion made of MiNET, and SEM images of array of MiNET micro-pillars. Silica $1 \mu \mathrm{m}$ particles were used in making hand skeleton and super activated nanoparticles were used for earth medallion and pillars. (b) Characterization of porous structure in the bulk of MiNET. SEM images of a micropillar on the base and the cross-section cut of the micropillar and its underneath base by FIB milling. (c) Confocal images of two MiNET micropillars: intact and broken. The intact pillar turns pink once infiltrated with dyed ethylene glycol (DEG). The part of the broken pillar attached to the base becomes pink due to infusion of DEG, but the rest of the part remains blue as DEG flow discontinues.

Table 1: Porosity and shrinkage data of different silica particles based MiNETs

\begin{tabular}{|c|c|c|c|c|}
\hline Samples & $\begin{array}{c}\text { Average Particle Size } \\
(\mu \mathrm{m})\end{array}$ & $\begin{array}{c}\text { Average Pore Dia. } \\
(\mu \mathrm{m})\end{array}$ & $\begin{array}{c}\text { Porosity } \\
(\%)\end{array}$ & $\begin{array}{c}\text { Shrinkage } \\
(\mathrm{V} / \mathrm{V} \%)\end{array}$ \\
\hline Silica $60 \mathrm{~nm}$ & 0.60 & 0.82 & 41.10 & 0.30 \\
\hline Silica $400 \mathrm{~nm}$ & 0.40 & 0.536 & 27.23 & 0.16 \\
\hline Silica $1 \mu \mathrm{m}$ & 1 & 1.142 & 25.03 & 0.14 \\
\hline Silica $10 \mu \mathrm{m}$ & 10 & 7.401 & 21.47 & 0.13 \\
\hline Silica $45 \mu \mathrm{m}$ & 45 & 11.120 & & \\
\hline
\end{tabular}




\section{References}

[1] M. Martina, G. Subramanyam, J. C. Weaver, D. W. Hutmacher, D. E. Morse, S. Valiyaveettil, Biomaterials 2005, 26, 5609.

[2] I. K. Sung, Christian, M. Mitchell, D. P. Kim, P. J. A. Kenis, Adv. Funct. Mater. 2005, 15,1336 .

[3] D. Liu, L. He, W. W. Lei, K. D. Klika, L. X. Kong, Y. Chen, Adv. Mater. Interfaces 2015, 2 .

[4] W. Y. Lv, Q. Q. Mei, J. L. Xiao, M. Du, Q. Zheng, Adv. Funct. Mater. 2017, 27.

[5] J. R. Wilson, W. Kobsiriphat, R. Mendoza, H. Y. Chen, J. M. Hiller, D. J. Miller, K.

Thornton, P. W. Voorhees, S. B. Adler, S. A. Barnett, Nat. Mat. 2006, 5, 541.

[6] C. A. L. Colard, R. A. Cave, N. Grossiord, J. A. Covington, S. A. F. Bon, Adv. Mater. 2009, 21, 2894.

[7] W. P. Lustig, S. Mukherjee, N. D. Rudd, A. V. Desai, J. Li, S. K. Ghosh, Chem. Soc. Rev. 2017, 46, 3242.

[8] D. Schneider, D. Mehlhorn, P. Zeigermann, J. Kärger, R. Valiullin, Chem. Soc. Rev. 2016, 45, 3439.

[9] Q.-L. Zhu, Q. Xu, Chem. Soc. Rev. 2014, 43, 5468.

[10] M. Destribats, B. Faure, M. Birot, O. Babot, V. Schmitt, R. Backov, Adv. Funct. Mater. 2012, 22, 2642.

[11] T. Ohji, M. Fukushima, Int. Mater. Rev. 2012, 57, 115.

[12] H. F. Zhang, A. I. Cooper, Soft Matter 2005, 1, 107.

[13] K. Stratford, R. Adhikari, I. Pagonabarraga, J. C. Desplat, M. E. Cates, Science 2005, 309, 2198.

[14] E. M. Herzig, K. A. White, A. B. Schofield, W. C. K. Poon, P. S. Clegg, Nat. Mat. 2007, 6,966 .

[15] D. Y. Cai, P. S. Clegg, Chem. Commun. 2015, 51, 16984.

[16] M. F. Haase, K. J. Stebe, D. Lee, Adv. Mater. 2015, 27, 7065.

[17] N. Tanaka, H. Kobayashi, N. Ishizuka, H. Minakuchi, K. Nakanishi, K. Hosoya, T.

Ikegami, Journal of Chromatography A 2002, 965, 35.

[18] Y. Ding, M. W. Chen, J. Erlebacher, J. Am. Chem. Soc. 2004, 126, 6876.

[19] B. H. Jones, T. P. Lodge, J. Am. Chem. Soc. 2009, 131, 1676.

[20] H. Y. Chen, Y. Kwon, K. Thornton, Scripta Mater. 2009, 61, 52.

[21] S. Torquato, S. Hyun, A. Donev, Phys. Rev. Lett. 2002, 89.

[22] H. G. Zhang, X. D. Yu, P. V. Braun, Nat. Nanotechnol. 2011, 6, 277.

[23] L. F. Wang, J. Lau, E. L. Thomas, M. C. Boyce, Adv. Mater. 2011, 23, 1524.

[24] J. H. Lee, L. F. Wang, M. C. Boyce, E. L. Thomas, Nano Lett. 2012, 12, 4392.

[25] M. E. Cates, P. S. Clegg, Soft Matter 2008, 4, 2132.

[26] M. N. Lee, A. Mohraz, Adv. Mater. 2010, 22, 4836.

[27] J. W. Tavacoli, J. H. J. Thijssen, A. B. Schofield, P. S. Clegg, Adv. Funct. Mater. 2011, 21,2020 .

[28] M. N. Lee, J. H. J. Thijssen, J. A. Witt, P. S. Clegg, A. Mohraz, Adv. Funct. Mater. 2013, 23, 417.

[29] M. N. Lee, M. A. Santiago-Cordoba, C. E. Hamilton, N. K. Subbaiyan, J. G. Duque, K. A. D. Obrey, J. Phys. Chem. Lett. 2014, 5, 809. 
[30] D. Y. Cai, P. S. Clegg, T. Li, K. A. Rumble, J. W. Tavacoli, Soft Matter 2017, 13, 4824.

[31] C. L. Huang, J. Forth, W. Y. Wang, K. L. Hong, G. S. Smith, B. A. Helms, T. P. Russell, Nat. Nanotechnol. 2017, 12, 1060.

[32] A. Gardziella, Pilato, L. A., Knop, A., Phenolic Resins: Chemistry, Applications, Standardization, Safety and Ecology, Springer, New York 2000.

[33] B. P. Binks, T. S. Horozov, Eds., Colloidal Particles at Liquid Interfaces, Cambridge University Press, Cambridge 2006.

[34] J. Forth, P. S. Clegg, Langmuir 2016, 32, 6387.

[35] H. S. Wi, S. Cingarapu, K. J. Klabunde, B. M. Law, Langmuir 2011, 27, 9979.

[36] R. Pal, AICHE J. 1996, 42, 3181.

[37] X.-Z. Wang, J. He, Y.-X. Weng, J.-B. Zeng, Y.-D. Li, Polym. Test. 2019, 79, 106057.

[38] Y.-D. Li, X.-Y. Jian, J. Zhu, A.-K. Du, J.-B. Zeng, Polym. Test. 2018, 72, 140.

[39] A. Mostafaei, F. Nasirpouri, Prog. Org. Coat. 2014, 77, 146.

[40] J. C. Fothergill, J. K. Nelson, M. Fu, Ieee, presented at 2004 Annual Report Conference on Electrical Insulation and Dielectric Phenomena, Boulder, CO, 2004.

[41] S. Singha, M. J. Thomas, IEEE Trans. Dielectr. Electr. Insul. 2009, 16, 531.

[42] J. R. Dixon, B. F. Marek, presented at SPE Annual Technical Conference and Exhibition, New Orleans, Louisiana, 1990/1/1/, 1990.

[43] X. Li, L. M. Zhang, Canadian Geotechnical Journal 2009, 46, 129.

[44] S. Sosnowski, P. Woźniak, M. Lewandowska-Szumieł, Macromol. Biosci. 2006, 6, 425.

[45] M. Peroglio, L. Gremillard, C. Gauthier, L. Chazeau, S. Verrier, M. Alini, J. Chevalier, Acta Biomater. 2010, 6, 4369.

[46] A. Peutzfeldt, Eur. J. Oral Sci. 1997, 105, 97. 


\section{Supporting Information}

\section{Shear-Induced Microporous Nanocomposite Epoxy Thermosets (MiNET)}

Molla Hasan, Yogin Patel, Arielle R. Gamboa, Michael J. Grzenda, Valeria Saro-Cortes, Vivek Mhatre, Jonathan P. Singer

Table SI1: Compositions of different MiNET prepared by direct mixing

\begin{tabular}{|l|c|c|c|c|c|c|}
\hline $\begin{array}{l}\text { Particles used to } \\
\text { make MiNET }\end{array}$ & $\begin{array}{c}\text { Average } \\
\text { particle size }\end{array}$ & $\begin{array}{c}\text { EPON 828 } \\
(\mathrm{gm})\end{array}$ & $\begin{array}{c}\text { Versamid 125 } \\
(\mathrm{gm})\end{array}$ & $\begin{array}{c}\text { ESO } \\
(\mathrm{gm})\end{array}$ & $\begin{array}{c}\text { Canola Oil } \\
(\mathrm{gm})\end{array}$ & $\begin{array}{c}\text { Particles } \\
\text { Amount } \\
(\mathrm{gm})\end{array}$ \\
\hline $\begin{array}{l}\text { Activated } \\
\text { Carbon }\end{array}$ & $150 \mathrm{~nm}$ & 0.40 & 0.40 & 0.20 & 2 & 2 \\
\hline Alumina & $20 \mathrm{~nm}$ & 0.40 & 0.40 & 0.20 & 2 & 4 \\
\hline $\begin{array}{l}\text { Super Activated } \\
\text { Carbon }\end{array}$ & $100 \mathrm{~nm}$ & 0.40 & 0.40 & 0.20 & 2 & 2 \\
\hline Zinc Oxide & $30 \mathrm{~nm}$ & 0.40 & 0.40 & 0.20 & 2 & 7.5 \\
\hline Chitosan & $25-30 \mu \mathrm{m}$ & 0.40 & 0.40 & 0.20 & 2 & 2.7 \\
\hline Graphene & $11-15 \mathrm{~nm}$ & 0.40 & 0.40 & 0.20 & 2 & 4 \\
\hline Graphite flakes & $1 \mu \mathrm{m}$ & 0.40 & 0.40 & 0.20 & 2 & 4 \\
\hline \multirow{5}{*}{ Silica } & $60 \mathrm{~nm}$ & 0.40 & 0.40 & 0.20 & 2 & 7.5 \\
\cline { 2 - 7 } & $400 \mathrm{~nm}$ & 0.40 & 0.40 & 0.20 & 2 & 7.5 \\
\cline { 2 - 7 } & $1 \mu \mathrm{m}$ & 0.40 & 0.40 & 0.20 & 2 & 7.5 \\
\cline { 2 - 7 } & $10 \mu \mathrm{m}$ & 0.40 & 0.40 & 0.20 & 2 & 7.5 \\
\cline { 2 - 7 } & $45 \mu \mathrm{m}$ & 0.40 & 0.40 & 0.20 & 2 & 7.5 \\
\hline Aluminum & $100-130 \mathrm{~nm}$ & 0.40 & 0.40 & 0.20 & 2 & 3.5 \\
\hline Silicone & $3 \mu \mathrm{m}$ & 0.40 & 0.80 & 0.20 & 2 & 2.5 \\
\hline
\end{tabular}

a) Aluminum nanoparticles based MiNET contains 0.1g carbon nanotubes. 

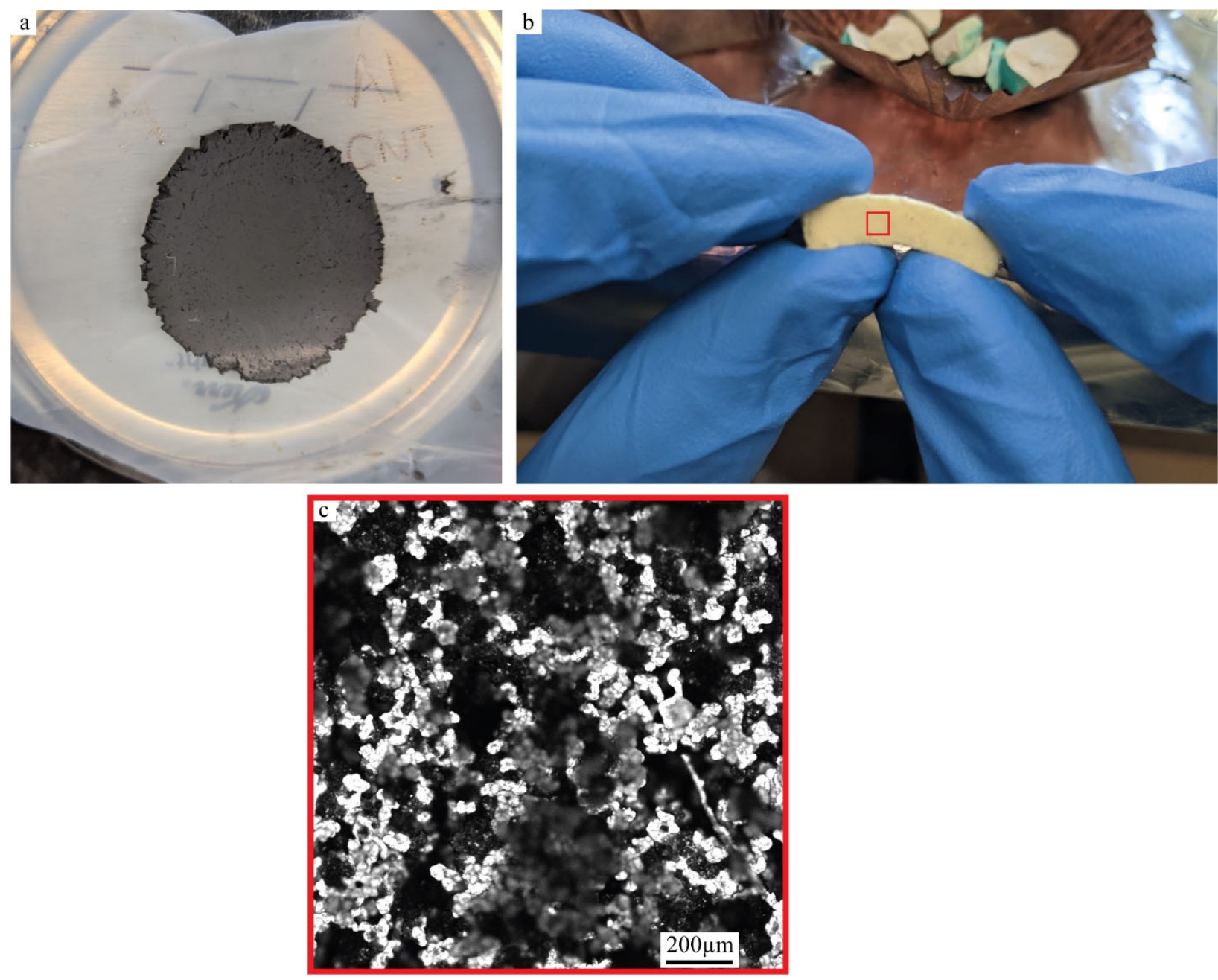

Figure SI1: (a) Aluminum nanoparticles based MiNET, (b) Flexible MiNET substrate using silicone microparticles, and (c) confocal image of the flexible MiNET. 